\title{
Hamiltonian Approach to Magnetic Fields with Toroidal Surfaces
}

A. Salat

Max-Planck-Institut für Plasmaphysik, EURATOM Association, Garching

Z. Naturforsch. 40 a, 959-967 (1985); received July 31, 1985

The equivalence of magnetic field line equations to a one-dimensional time-dependent Hamiltonian system is used to construct magnetic fields with arbitrary toroidal magnetic surfaces $I=$ const. For this purpose Hamiltonians $H$ which together with their invariants satisfy periodicity constraints have to be known. The choice of $H$ fixes the rotational transform $\eta(I)$. Arbitrary axisymmetric fields, and nonaxisymmetric fields with constant $\eta(I)$ are considered in detail.

Configurations with coinciding magnetic and current density surfaces are obtained. The approach used is not well suited, however, to satisfying the additional MHD equilibrium condition of constant pressure on magnetic surfaces.

\section{Introduction}

In plasma physics the topology of magnetic fields is of particular importance. The magnetohydrodynamic equilibrium equation for the current density $\boldsymbol{j}$ and the magnetic field $\boldsymbol{B}$,

$$
\boldsymbol{j} \times \boldsymbol{B}=\nabla p,
$$

requires that the field lines be confined to toroidal nested surfaces $I=$ const, provided the pressure $p$ is constant on $I=$ const surfaces and has a finite normal gradient.

It is well known [1], [2], [3] that the field line equations can be written as a one-dimensional time-^ dependent Hamiltonian system. An integrable Hamiltonian corresponds to the existence of magnetic surfaces. The aim of the paper is to explore to what extent the Hamiltonian structure may be used to construct magnetic fields with desired properties.

In Sect. 2 the field line Hamiltonian is recapitulated. It is shown that a magnetic field with arbitrary magnetic surfaces $I=$ const can always be obtained from any suitable integrable Hamiltonian. The Hamiltonian alone determines the rotational transform $\eta(I)$. The general axisymmetric field, a particular class of nonaxisymmetric fields with constant rotational transform, and the combination of the two classes are considered in detail.

The necessary equilibrium condition that the magnetic surfaces and the current density surfaces

Reprint requests to Dr. A. Salat, Max-Planck-Institut für Plasmaphysik, EURATOM Association, 8046 Garching.
$J=$ const coincide is discussed in Sect. 3, and appropriate configurations for the examples mentioned are presented. In Sect. 4 it is attempted to satisfy all three components of (1.1). It turns out that the particular Hamiltonian approach used is not very suitable for this purpose. Conclusions are given in Section 5 .

\section{Magnetic Surfaces}

Let $r^{1}, r^{2}, r^{3}$ be an arbitrary nonsingular coordinate system, such that $r^{2}$ and $r^{3}$ are a poloidal and a toroidal angle, respectively, with periodicity $2 \pi$. Covariant and contravariant components of any vector $\boldsymbol{A}=A^{i} \boldsymbol{e}_{i}=A_{i} \nabla r^{i}$ (with summation convention) are defined by

$A_{i}=\boldsymbol{A} \cdot \boldsymbol{e}_{i} \quad$ and $\quad \boldsymbol{A}^{i}=\boldsymbol{A} \cdot \nabla r^{i}, \quad i=1,2,3$,

respectively, where $\boldsymbol{e}_{i}=\partial \boldsymbol{r} / \partial r^{i}, \boldsymbol{r}=$ position vector. The usual relations

$$
\begin{aligned}
& A^{i}=g^{i j} A_{j}, \quad A_{i}=g_{i j} A^{j}, \\
& g^{i j}=\nabla r^{i} \cdot \nabla r^{j}, \quad g_{i j}=\boldsymbol{e}_{i} \cdot \boldsymbol{e}_{j}, \\
& \sqrt{g}=\left(\left[\nabla r^{1} \times \nabla r^{2}\right] \cdot \nabla r^{3}\right)^{-1}
\end{aligned}
$$

will also be used.

Let $\boldsymbol{A}$ be the vector potential of the magnetic field $\boldsymbol{B}$. The relation $\boldsymbol{B}=\operatorname{curl} \boldsymbol{A}$, which implies $\operatorname{div} \boldsymbol{B}=0$, in component notation is

$$
B^{1}=\frac{1}{\sqrt{g}}\left(\frac{\partial A_{3}}{\partial r^{2}}-\frac{\partial A_{2}}{\partial r^{3}}\right),
$$


etc., with $B^{2}, B^{3}$ from cyclic permutation of (2.3). To the vector potential $\boldsymbol{A}$ one can always add the gradient of an arbitrary function without changing $\boldsymbol{B}$. This can be used to obtain $A_{1}=0$. With this gauge one obtains

$$
B^{2}=\frac{-1}{\sqrt{g}} \frac{\partial A_{3}}{\partial r^{1}}, \quad B^{3}=\frac{1}{\sqrt{g}} \frac{\partial A_{2}}{\partial r^{1}} .
$$

If the angle $r^{3}$ is used as a parameter along the field lines, the equations for the field lines are

$$
\frac{\mathrm{d} r^{1}}{\mathrm{~d} r^{3}}=\frac{B^{1}}{B^{3}}, \quad \frac{\mathrm{d} r^{2}}{\mathrm{~d} r^{3}}=\frac{B^{2}}{B^{3}} .
$$

The equations are equivalent to a one-dimensional time-dependent Hamiltonian system [1], [2] if the following identifications are made:

$$
\begin{aligned}
& p=-A_{2}\left(r^{1}, r^{2}, r^{3}\right), \quad q=r^{2}, \quad t=r^{3}, \\
& H=A_{3}\left(r^{1}, r^{2}, r^{3}\right)
\end{aligned}
$$

(a confusion of the momentum $p$ with the pressure $p(I)$ should not occur). Consider, for example, $\dot{q}=\partial H / \partial p$. From (2.6) and (2.7) it follows that

$$
1=-\frac{\partial A_{2}}{\partial p}=-\frac{\partial A_{2}}{\partial r^{1}} \frac{\partial r^{1}}{\partial p}
$$

and

$$
\dot{q}=\frac{\partial A_{3}}{\partial p}=\frac{\partial A_{3}}{\partial r^{1}} \frac{\partial r^{1}}{\partial p},
$$

so that

$$
\dot{q}=\frac{\mathrm{d} r^{2}}{\mathrm{~d} r^{3}}=-\frac{\partial A_{3}}{\partial r^{1}} / \frac{\partial A_{2}}{\partial r^{1}}=\frac{B^{2}}{B^{3}}
$$

which is the second part of (2.5). The first part follows analogously.

Hamiltonians $H(p, q, t)$ are always integrable in the sense that they possess, in general unknown, single-valued invariants $I_{H}(p, q, t)$, such that

$$
\dot{I}_{H}=\frac{\partial I_{H}}{\partial p} \dot{p}+\frac{\partial I_{H}}{\partial q} \dot{q}+\frac{\partial I_{H}}{\partial t}=0 .
$$

This follows from the fact that the initial values $p_{0}$ and $q_{0}$ at $t=t_{0}$ are related to $p$ and $q$ by a succession of local nonsingular canonical transformations [4]. They therefore represent invariants $I_{H}(p, q, t)$.

If $p$ is replaced by $-A_{2},(2.6)$, one obtains invariant surfaces $I\left(r^{1}, r^{2}, r^{3}\right)=$ const in configuration space:

$$
I_{H}(p, q, t)=I_{H}\left(p\left(r^{1}, r^{2}, r^{3}\right), r^{2}, r^{3}\right) \equiv I\left(r^{1}, r^{2}, r^{3}\right) .
$$

These invariant surfaces are magnetic surfaces. This follows from (2.5) (if it is assumed that $B^{3} \neq 0$ ):

$\dot{I}=\frac{\partial I}{\partial r^{1}} \dot{r}^{1}+\frac{\partial I}{\partial r^{2}} \dot{r}^{2}+\frac{\partial I}{\partial r^{3}} \dot{r}^{3}=\frac{1}{B^{3}} \boldsymbol{B} \cdot \nabla I=0$.

In general, however, the invariant surfaces, apart from being unknown, are unphysical because the invariants $I_{H}$ will not be $(2 \pi$-)periodic and hence not single-valued in the angles $r^{2}=q, r^{3}=t$.

So, for a given magnetic field its vector potential in the proper gauge defines a corresponding Hamiltonian system, but in most cases one cannot decide whether it is "truly" integrable, i.e. whether the field has single-valued magnetic surfaces. It is possible, however, to reverse the roles of given and of desired quantities and to use the Hamiltonian formulation for the construction of magnetic fields with desired magnetic surfaces. This can be done in the following way:

Two functions have to be specified to define the relation between the magnetic field and the Hamiltonian system. As will be shown presently, one can choose a Hamiltonian $H(p, q, t)$ and the function $I\left(r^{1}, r^{2}, r^{3}\right)$, which defines the magnetic surfaces $I=$ const, provided an invariant $I_{H}(p, q, t)$ of $H$ is known. Different Hamiltonians correspond to different magnetic fields but with the same magnetic surfaces. Knowledge of time-dependent Hamiltonians with known invariant(s) has steadily increased in recent years (see, for example, [5]-[8]). Both the Hamiltonians and the invariants have to be such that the resulting magnetic field is periodic in $r^{2}$ and $r^{3}$.

With $H(p, q, t)$ given and an invariant $I_{H}(p, q, t)$ known, $I_{H}$ is equal to the desired function $I$,

$$
I_{H}\left(p, r^{2}, r^{3}\right)=I\left(r^{1}, r^{2}, r^{3}\right) \text {. }
$$

This defines $p$ as a function of $r^{1}, r^{2}, r^{3}$ and hence defines the vector potential $A_{2}=-p,(2.6)$. Furthermore, from (2.7) one gets

$$
A_{3}\left(r^{1}, r^{2}, r^{3}\right)=H\left(p\left(r^{1}, r^{2}, r^{3}\right), r^{2}, r^{3}\right),
$$

so that $A_{1}=0, A_{2}$ and $A_{3}$, i.e. the magnetic field, is indeed determined by prescribing its magnetic surfaces and a Hamiltonian with known invariant. Examples are given below. The method is only practicable provided (2.14) can be solved explicitly for $p$. This rules out Hamiltonians with invariants of too complicated structure. 
It is also possible to express the vector potentials $A_{i}$ completely in terms of the magnetic surface label I (instad of $r^{1}$ ) and the angles $r^{2}, r^{3}$. This follows from (2.14), which can be written as

$$
I_{H}\left(-A_{2}, r^{2}, r^{3}\right)=I
$$

and defines $A_{2}=\tilde{A}_{2}\left(I, r^{2}, r^{3}\right)$. Similarly, one has

$$
A_{3}=\tilde{A}_{3}\left(I, r^{2}, r^{3}\right)=H\left(-\tilde{A}_{2}, r^{2}, r^{3}\right),
$$

where the tilde indicates the dependence on the surface label. The poloidal and toroidal fluxes $\Phi_{p}$ and $\Phi_{t}$ are then easily found:

$$
\begin{aligned}
\Phi_{p}(I) & =\iint \mathrm{d} r^{1} \mathrm{~d} r^{3} \sqrt{g} B^{2} \\
& =-\oint \mathrm{d} r^{3} \tilde{A}_{3}\left(I, r^{2}, r^{3}\right), \\
\Phi_{t}(I) & =\iint \mathrm{d} r^{1} \mathrm{~d} r^{2} \sqrt{g} B^{3} \\
& =\oint \mathrm{d} r^{2} \tilde{A}_{2}\left(I, r^{2}, r^{3}\right) .
\end{aligned}
$$

It can be shown that the dependence of $\Phi_{p}$ and $\Phi_{t}$ on $r^{2}$ and $r^{3}$, respectively, is only apparent, as it of course must be.

The rotational transform $\eta(I)$ is defined by

$$
\eta(I)=\Phi_{p}^{\prime}(I) / \Phi_{t}^{\prime}(I)
$$

where the prime denotes differentiation with respect to $I$. It therefore follows that prescribing the Hamiltonian of a magnetic field is equivalent to prescribing its rotational transform. We shall come back to this point during and after the discussion of several examples.

\section{Example 1: axisymmetric fields}

In the axisymmetric case the Hamiltonian and the invariant surfaces are independent of $t=r^{3}$. Hence, the Hamiltonian itself is an invariant:

$$
I_{H}(p, q)=H(p, q) .
$$

One can choose any Hamiltonian $H(p, q)$ and any desired function $I\left(r^{1}, r^{2}\right)$ for the magnetic surfaces $I=$ const and one immediately obtains the vector potential from

$$
H\left(-A_{2}, r^{2}\right)=I\left(r^{1}, r^{2}\right), \quad A_{3}=I\left(r^{1}, r^{2}\right) .
$$

For $H(p, q) 2 \pi$-periodic in $q$ the magnetic field is automatically periodic in $r^{2}$, because the potentials are. According to (2.4) only the derivative $\partial A_{2} / \partial r^{1}$ need be periodic. This also allows a more general dependence of $H(p, q)$ on $q$.
The magnetic field is obtained from (2.3), (2.4) and (2.21):

$$
\begin{aligned}
& B^{1}=\frac{1}{\sqrt{g}} \frac{\partial I}{\partial r^{2}}, \quad B^{2}=\frac{-1}{\sqrt{g}} \frac{\partial I}{\partial r^{1}}, \\
& B^{3}=\frac{-1}{\sqrt{g}}\left(\frac{\partial H}{\partial p}\right)^{-1} \frac{\partial I}{\partial r^{1}} .
\end{aligned}
$$

The magnetic fluxes follow from (2.18) and

$$
H\left(-\tilde{A}_{2}, r^{2}\right)=I, \quad \tilde{A}_{3}=I
$$

which imply $\Phi_{p}=-2 \pi I$. Differentiation of (2.23) yields the rotational transform

$$
\eta(I)=\frac{2 \pi}{\oint \mathrm{d} r^{2}\left(\frac{\partial H}{\partial p}\right)^{-1}}=\frac{2 \pi}{\oint \mathrm{d} r^{2} \frac{B^{3}}{B^{2}}},
$$

where $\partial H / \partial p$ or $B^{2}$ and $B^{3}$ have to be expressed in terms of $I$ and $r^{2}$. By properly choosing $H(p, q)$ the rotational transform may be given any desired dependence on $I$.

Example 2: nonaxisymmetric shear-free fields

We consider the special class of time-dependent Hamiltonians

$$
H(p, q, t)=p G(q, t),
$$

where $G$ is $2 \pi$-periodic in both arguments. Invariants of $H$ are assumed to have the form

$$
I_{H}(p, q, t)=p K(q, t),
$$

where $K$ is also $2 \pi$-periodic in both arguments. $I\left(r^{1}, r^{2}, r^{3}\right)$ may again be arbitrary ( $2 \pi$-periodic).

A trivial example of the combined structure of (2.25), (2.26), case $A$, is

$G(q, t)=G_{1}(q) G_{2}(t), \quad K(q, t)=G_{1}(q)$,

where $G_{1}$ and $G_{2}$ are arbitrary periodic functions. Another, nontrivial example [5], [6], case $B$, is

$G(q, t)=1+\left[\omega^{2}(t)-1\right] \cos ^{2} q$,

$K(q, t)=\frac{1}{R^{2}(t)} \cos ^{2} q+(\dot{R} \cos q-R \sin q)^{2}$,

where $R(t)$ is an arbitrary periodic function and

$$
\omega^{2}(t)=\frac{1}{R^{4}}-\frac{\ddot{R}}{R} .
$$

Not very much is known about integrals of $H=p G(q, t)$ where $G$ is not related to cases $A$ or $B$. Preliminary results indicate, for example, that 
generically $K(q, t)$ contains infinitely many harmonics $e^{i m q}$ if $G$ contains more than two of them. In numerical studies related to [9] using Poincaré surface-of-section cuts, integrability with periodic invariants was found for "most" randomly selected functions $G(q, t)$ in finite parameter intervalls. Hamiltonians of type (2.25), chosen for their formal simplicity, are of a special type, the non-KAM type [9] (see Appendix). This, however, does not affect the subsequent qualitative conclusions.

From $\dot{I}=(p K)^{\circ}=0$ it is straightforward to derive the equation

$$
\frac{\partial}{\partial q} \frac{G}{K}+\frac{\partial}{\partial t} \frac{1}{K}=0 \text {. }
$$

Combining (2.25), (2.26), (2.14), (2.15) and (2.3), (2.4), (2.29) yields

$$
\begin{aligned}
& B^{1}=\frac{1}{\sqrt{g}} \frac{1}{K}\left(G \frac{\partial I}{\partial r^{2}}+\frac{\partial I}{\partial r^{3}}\right), \\
& B^{2}=\frac{-1}{\sqrt{g}} \frac{G}{K} \frac{\partial I}{\partial r^{1}}, \\
& B^{3}=\frac{-1}{\sqrt{g}} \frac{1}{K} \frac{\partial I}{\partial r^{1}} .
\end{aligned}
$$

As a consequence, the magnetic field is expressed completely in terms of arbitrary surfaces $I\left(r^{1}, r^{2}, r^{3}\right)$ $=$ const, of $G\left(r^{2}, r^{3}\right)$ and of $K\left(r^{2}, r^{3}\right)$.

The rotational transform $\eta$ is found to be

$$
\eta=\oint \mathrm{d} r^{3} \frac{G}{K} / \oint \mathrm{d} r^{2} \frac{1}{K} .
$$

This is evidently independent of the surface $I$, so that Hamiltonians of type (2.25), (2.26) correspond to shear-free magnetic fields as approximately realized in heliotron and stellarator configurations [10]. Equation (2.29) ensures that $\eta$ is a true surface function.

For the special examples (2.27 A), (2.27 B) one easily obtains

$$
\begin{aligned}
& \eta=\left\langle G_{2}\right\rangle /\left\langle 1 / G_{1}\right\rangle, \\
& \eta=\left\langle 1 / R^{2}\right\rangle,
\end{aligned}
$$

where \langle\rangle denotes the average with respect to $r^{2}$ or $r^{3}$.

Evample 3: axisymmetric shear-free fields

This case, with

$$
H(p, q)=p G(q),
$$

$G(q) 2 \pi$-periodic, is a combination of examples 1 and 2. It is unnecessary to repeat the results for $B^{i}$ and $\eta(I)$ which simply follow from (2.30), (2.31) by setting $K\left(r^{2}, r^{3}\right)=G\left(r^{2}, r^{3}\right)=G\left(r^{2}\right)$. Rather, the example is chosen to direct attention to the following point which is relevant below:

Looking for axisymmetric configurations with constant rotational transform $\eta(I)$, one obtains from (2.18), (2.19) and (2.23)

$$
\left\langle\frac{\partial \tilde{A}_{2}\left(I, r^{2}\right)}{\partial I}\right\rangle=-\frac{1}{\eta}
$$

which is satisfied by

$$
\tilde{A}_{2}\left(I, r^{2}\right)=-I M\left(r^{2}\right)+N\left(I, r^{2}\right),
$$

provided $\left\langle M\left(r^{2}\right)\right\rangle=\eta^{-1}$ and

$$
\left\langle N\left(I, r^{2}\right)\right\rangle=0 .
$$

With $A_{2}=-p$ and $H=I$, one finally obtains

$$
H(p, q)=p \frac{1}{M(q)}-\frac{N(H, q)}{M(q)} .
$$

This shows that $H=p G(q)$ is far from being the most general Hamiltonian with constant rotational transform, $\left(G=M^{-1}\right)$. There is an arbitrary function $N(H, q)$, apart from condition (2.36), which yields all sorts of Hamiltonians with different structure. This is evidently a general result: If one picks an arbitrary class of Hamiltonians $H(p, q, t)$, the rotational transform is fixed but, generically, the Hamiltonians selected are not the most general ones with the same $\eta(I)$.

\section{Current Density Surfaces}

The MHD equation (1.1) implies that the magnetic fields discussed so far can only be in equilibrium with a finite pressure gradient provided the currents are confined to the same surfaces $I=$ const as the field lines are.

The existence and properties of current density surfaces $J=$ const can be treated, in principle, in the same way as that of magnetic surfaces. The lines of current

$$
\frac{\mathrm{d} r^{1}}{\mathrm{~d} r^{3}}=\frac{j^{1}}{j^{3}}, \quad \frac{\mathrm{d} r^{2}}{\mathrm{~d} r^{3}}=\frac{j^{2}}{j^{3}}
$$

may again be written as a conjugate pair of Hamiltonian equations provided $\operatorname{div} \boldsymbol{j}=0$ and

$$
B_{1}=0 \text {. }
$$


While $\operatorname{div} \boldsymbol{j}=0$ is automatically satisfied with $\boldsymbol{j}=\operatorname{curl} \boldsymbol{B}$, the second condition, which in the case of field lines is a simple condition on the gauge of the vector potential, is nontrivial. In order to satisfy it, one has to abandon the arbitrariness of the coordinate system: if one chooses $r^{1}$ as a function of the surface label I only, and the surfaces $r^{2}, r^{3}=$ const orthogonal to $I=$ const, i.e.

$$
g^{12}=g^{13}=0,
$$

then $\boldsymbol{B} \cdot \nabla I=0$ implies $B^{1}=B_{1}=0$. Such adapted coordinates will be used in the following except where otherwise stated (examples 1 and 3 below).

By analogy with (2.6), (2.7) the canonical coordinates $Q, P$ and the Hamiltonian $H_{\mathrm{c}}$ will be defined as

$$
\begin{aligned}
& P=-B_{2}\left(r^{1}, r^{2}, r^{3}\right), \quad Q=r^{2}, \quad t=r^{3}, \\
& H_{\mathrm{c}}=B_{3}\left(r^{1}, r^{2}, r^{3}\right) .
\end{aligned}
$$

$B_{2}$ and $B_{3}$ are not arbitrary because magnetic surfaces have been assumed to exist. Nevertheless, the Hamiltonian system (3.4), (3.5) is still so general that the existence of invariant surfaces $J=$ const is open. For this reason we again restrict further discussion to the examples $1-3$ of the previous section.

\section{Example 1:}

In the axisymmetric case the Hamiltonian $H_{\mathrm{c}}$ is also independent of $t$, and $H_{\mathrm{c}}(P, Q)$ itself is an invariant $J_{H}(P, Q)$. From (3.5) and by analogy with (2.21) an invariant in configuration space is

$$
J\left(r^{1}, r^{2}\right)=B_{3} .
$$

The equilibrium condition that the surfaces of field lines $I=$ const and of current density lines $J=$ const coincide therefore requires that

$$
B_{3}=F(I),
$$

where $F(I)$ so far is an arbitrary function. This condition also follows direct from $\boldsymbol{j} \cdot \nabla I=0, \boldsymbol{j}=\operatorname{curl} \boldsymbol{B}$ and axisymmetry, which proves that it is valid for arbitrary coordinates to which we revert in this example and example 3.

Equations (2.22) and (3.7) for the magnetic field can be combined into the classical form [11], [12]

$$
\boldsymbol{B}=\left[\nabla I \times \nabla r^{3}\right]+F(I) \nabla r^{3} .
$$

From (3.7), the third of eqs. (2.22) and axisymmetry one obtains

$$
F(I)=\frac{-1}{\sqrt{g} g^{33}} \frac{1}{\frac{\partial H}{\partial p}} \frac{\partial I\left(r^{1}, r^{2}\right)}{\partial r^{1}},
$$

where $\partial H / \partial p$ is to be understood at $p=-\tilde{A}_{2}\left(I, r^{2}\right)$ or $p=-A_{2}\left(r^{1}, r^{2}\right)$. Given $H(p, q)$ and $F(I)$, this differential equation determines the shape of the magnetic surfaces $I\left(r^{1}, r^{2}\right)=$ const. It is unexpected that the magnetic surfaces are already determined (up to free functions which appear in the integration procedure) from the condition that the current density surfaces coincide with $I=$ const. In Sect. 4 the relation with MHD equilibrium surfaces will be discussed.

From (2.24) and the poloidal average of (3.9) another relation may be derived,

$$
\begin{aligned}
F(I) & =\frac{1}{\eta(I)} \frac{-1}{\left\langle\sqrt{g} g^{33} /\left(\partial I / \partial r^{1}\right)\right\rangle} \\
& =\frac{1}{\eta(I)} \frac{1}{\left\langle g^{\left.33 / B^{2}\right\rangle}\right.}
\end{aligned}
$$

which shows that $F(I)$ is determined by the shape of the magnetic surfaces and the rotational transform. This relation is more general than (3.9) because it does not contain $H(p, q)$ explicitly. It is therefore valid for all $H(p, q)$ which have the same $\eta(I)$ (see Sect. 2, example 3). Relation (3.9b) is useful in the discussion of flux-conserving tokamak equilibria [13].

\section{Example 2:}

From (2.30), (3.4) and (3.5), and keeping in mind the special coordinate system introduced above, one obtains

$$
\begin{aligned}
& P=\left(g_{22} G+g_{23}\right) \frac{1}{\sqrt{g}} \frac{1}{K} \frac{\mathrm{d} I}{\mathrm{~d} r^{1}}, \\
& H_{\mathrm{c}}=-\left(g_{23} G+g_{33}\right) \frac{1}{\sqrt{g}} \frac{1}{K} \frac{\mathrm{d} I}{\mathrm{~d} r^{1}},
\end{aligned}
$$

so that the Hamiltonian for the current density may be written as

$$
H_{\mathrm{c}}(P, Q, t)=P \Gamma(P, Q, t),
$$

where

$$
\Gamma(P, Q, t)=-\frac{g_{23} G\left(r^{2}, r^{3}\right)+g_{33}}{g_{22} G\left(r^{2}, r^{3}\right)+g_{23}} .
$$


The dependence of $\Gamma$ on $r^{1}, r^{2}, r^{3}$ is determined by $G\left(r^{2}, r^{3}\right)$ and the surfaces $I=$ const. Inversion of (3.11) for $r^{1}=r^{1}\left(P, r^{2}, r^{3}\right)$ leads to $\Gamma(P, Q, t)$. In general, however, this cannot be done explicitly.

In order to proceed further, we consider the hypothetical case

$$
\Gamma(P, Q, t)=\Gamma(Q, t) .
$$

As a consequence,

$$
H_{\mathrm{c}}(P, Q, t)=P \Gamma(Q, t)
$$

would have the same structure as $H$ itself, viz. (2.25), and the results of Sect. 2 could therefore be applied to the current surfaces as well.

The question is, can condition (3.15) be satisfied? A sufficient, purely geometric condition is

$$
g_{23}=0
$$

together with

$$
\frac{\partial}{\partial r^{1}} g_{22}\left(r^{1}, r^{2}, r^{3}\right)=\frac{\partial}{\partial r^{1}} g_{33}\left(r^{1}, r^{2}, r^{3}\right)=0 .
$$

From $(\mathrm{d} r)^{2}=g_{i k} \mathrm{~d} r^{i} \mathrm{~d} r^{k}$ it follows that the arc lengths $L_{p}$ and $L_{t}$ of poloidally and toroidally closed coordinate lines $r^{1}, r^{3}=$ const and $r^{1}, r^{2}=$ const are given by

$$
L_{p}=\oint \mathrm{d} r^{2} \sqrt{g_{22}}, \quad L_{t}=\oint \mathrm{d} r^{3} \sqrt{g_{33}},
$$

respectively. Equations (3.18) therefore require that the lengths $L_{p}\left(r^{1}, r^{3}\right)$ and $L_{t}\left(r^{1}, r^{2}\right)$ be the same from one surface $r^{1}=I$ to the next. It should be possible to satisfy this requirement by the following scheme:

Starting from a relatively smooth toroidal surface, the successive surfaces towards the inside are progressively corrugated, both in the poloidal and toroidal cross-sections, in such a way that the lengths of the coordinate lines stay constant. This procedure can be continued until the corrugated surfaces reach the magnetic axis or close the hole of the torus. Obviously, there is considerable freedom in the configuration, stemming from the arbitrariness of the initial surface. It should be possible to select it such that the coordinate surfaces are mutually orthogonal in order to satisfy (3.17).

Having "constructed" nested sets of surfaces destined to become current density surfaces $J=$ const, one is free to use them as magnetic surfaces $I=$ const as well, setting

$$
J=F(I),
$$

with $F(I)$ arbitrary. As a final problem, $G\left(r^{2}, r^{3}\right)$ has to be found such that not only does $H=p G(q, t)$ have doubly periodic invariants but also

$$
H_{\mathrm{c}}=-P \frac{g_{33}(Q, t)}{G(Q, t) g_{22}(Q, t)} .
$$

According to the experience gained in [9] this should be possible.

This requires, however, that an explicit representation of the corrugated surfaces together with the proper coordinate system be at hand.

\section{Example 3:}

With $G\left(r^{2}, r^{3}\right)=K\left(r^{2}, r^{3}\right)=G\left(r^{2}\right)$ and (2.31) the relation ( 3.10 a) between $F(I)$ and $I$ becomes

$$
F(I)=\langle 1 / G\rangle \frac{-1}{\left\langle\sqrt{g} g^{33} /\left(\partial I / \partial r^{1}\right)\right\rangle} .
$$

On the other hand, for given $F(I)$ and $H=p G(q)$ the differential equation (3.9) may be integrated explicitly to give the family of magnetic surfaces

$$
I\left(r^{1}, r^{2}\right)=f\left(I_{0}\left(r^{2}\right)+G\left(r^{2}\right) c\left(r^{1}, r^{2}\right)\right),
$$

where $I_{0}\left(r^{2}\right)$ is an arbitrary periodic function,

$$
c\left(r^{1}, r^{2}\right)=\int_{0}^{r^{1}} \mathrm{~d} r^{1} \sqrt{g} g^{33},
$$

and the inverse of $f$ is defined by

$$
f^{-1}(I)=-\int_{0}^{I} \frac{\mathrm{d} I}{F(I)} .
$$

The radial integration in (3.24) may be performed in, for example, orthogonal coordinates which are related to Cartesian coordinates $x, y, z$ by

$x=R \cos r^{3}, \quad y=R \sin r^{3}, \quad z=r^{1} \sin r^{2}$,

where $R=R_{0}-r^{1} \cos r^{2}$, and $R_{0}$ is the radius of the circular magnetic axis. With $\sqrt{g}=r^{1} R$, and $g^{33}=$ $1 / R^{2}$ one obtains

$c\left(r^{1}, r^{2}\right)=\frac{-1}{\cos ^{2} r^{2}}\left[r^{1} \cos r^{2}+R_{0} \ln \left(1-\frac{r^{1}}{R_{0}} \cos r^{2}\right)\right]$.

For $\left|r^{1} \cos r^{2}\right| \ll R_{0}, \quad c\left(r^{1}, r^{2}\right) \cong\left(r^{1}\right)^{2} /\left(2 R_{0}\right)$ stays bounded. For $f, I_{0}$ and $G \neq 0$ the surfaces $I=$ const are nested around the magnetic axis, bounded, and crowded together close to the axis of symmetry.

Again, discussion of (3.23) is deferred to the next section. 


\section{MHD Equilibrium Pressure Balance}

In the last two sections we have either derived or made plausible the existence of configurations with coinciding nested surfaces $I\left(r^{1}, r^{2}, r^{3}\right)=$ const of field lines and current density. This, however, is not sufficient for the existence of MHD equilibria with surfaces of constant pressure. The coinciding surfaces imply (in arbitrary coordinates)

$$
[\boldsymbol{j} \times \boldsymbol{B}]=a\left(r^{1}, r^{2}, r^{3}\right) \nabla I,
$$

where $a\left(r^{1}, r^{2}, r^{3}\right)$ depends on the details of the field. According to (1.1) however, with $p=p(I)$, the function $a$ has to depend on the coordinates via $I\left(r^{1}, r^{2}, r^{3}\right)$ only:

$$
a=\frac{\mathrm{d} p(I)}{\mathrm{d} I} .
$$

This yields an additional condition. In axisymmetry it is the Grad-Shafranov condition [11], [12] (4.4). In order to discuss the equilibrium condition, we gain turn to the previously used examples.

\section{Examples 1 and 3:}

The first component of the equilibrium relation is

$$
(\nabla p)_{1}=\frac{\mathrm{d} p}{\mathrm{~d} I} \frac{\partial I}{\partial r^{1}}=\sqrt{g}\left(j^{2} B^{3}-j^{3} B^{2}\right) .
$$

$B^{3}$ may be expressed in terms of $F(I),(3.7)$, while all other quantities may be expressed in terms of derivatives of $I\left(r^{1}, r^{2}\right)$ via the first two of (2.22). The result is the Grad-Shafranov equation

$$
\begin{aligned}
& \frac{\partial}{\partial r^{1}}\left(\frac{1}{\sqrt{g} g^{22}} \frac{\partial I}{\partial r^{1}}\right)+\frac{\partial}{\partial r^{2}}\left(\frac{1}{\sqrt{g} g^{11}} \frac{\partial I}{\partial r^{2}}\right) \\
& +\sqrt{g} \frac{\mathrm{d} p}{\mathrm{~d} I}+\sqrt{g} g^{33} F(I) \frac{\mathrm{d} F}{\mathrm{~d} I}=0,
\end{aligned}
$$

written here for arbitrary orthogonal coordinates $r^{1}, r^{2}$, with $r^{3}$ being the angle around the axis of symmetry. The two profiles $p(I)$ and $F(I)$ have to be specified in order to determine the magnetic surfaces $I\left(r^{1}, r^{2}\right)=$ const.

In Sect. 3, on the other hand, magnetic surfaces have been determined from $H(p, q)$ and $F(I)$ merely by the requirement that the magnetic and current density surfaces be identical. The question is whether these " $B j$-surfaces" are always contained among the "GS-surfaces" (Grad-Shafranov) with some pressure profile $p(I)$ to be determined a posteriori.
This can be tested in example 3 with the $B j$-surfaces given explicitly in (3.23). Inserting $I\left(r^{1}, r^{2}\right)$ in (4.4) yields

$$
\begin{aligned}
\frac{\mathrm{d} p}{\mathrm{~d} I}= & -F \frac{\mathrm{d} F}{\mathrm{~d} I}\left\{G^{2} \frac{\left(r^{1}\right)^{2}}{R^{4}}+\frac{1}{\left(r^{1} R\right)^{2}}\right. \\
& \left.\cdot\left[\frac{\partial}{\partial r^{2}}\left(I_{0}+G c\right)\right]^{2}+\frac{1}{R^{2}}\right\}+F \frac{1}{r^{1} R} \\
& \cdot\left\{G \frac{\partial}{\partial r^{1}}\left(\frac{r^{1}}{R}\right)^{2}+\frac{\partial}{\partial r^{2}}\left[\frac{1}{r^{1} R} \frac{\partial}{\partial r^{2}}\left(I_{0}+G c\right)\right]\right\},
\end{aligned}
$$

where $G\left(r^{2}\right), I_{0}\left(r^{2}\right)$ are the only free functions. Owing to the given dependence of the r.h.s. on $r^{1}$, and there being no free function of $r^{1}$ available, it is obviously impossible in general to turn the r.h.s. into a function of $I\left(r^{1}, r^{2}\right)$ alone by any nontrivial choice of $G$ and $I_{0}$. In consequence, it is impossible in general to proceed from $B j$-surfaces to $G S$-surfaces if the $B j$-surfaces are derived from an arbitrary pair of functions $H(p, q), F(I)$.

The reason for this result is easy to understand. If instead of the differential (3.9) its poloidal average, (3.10 a), is used to express $F(I)$ in terms of $I\left(r^{1}, r^{2}\right)$, the $G S$-equation becomes an integro-differential equation for $I\left(r^{1}, r^{2}\right)$. The profile $\eta(I)$ has to be specified, with $\eta(I)=$ const in example 3 . The solution $I\left(r^{1}, r^{2}\right)$ in turn determines $F(I),(3.10 \mathrm{a})$, so that the differential equation (3.9) finally serves to determine $(\partial H / \partial p)^{-1}$. More precisely, since the averaged equation has already been satisfied, the poloidal variation $\delta(\partial H / \partial p)^{-1}$ is determined, where $\delta A=A-\langle A\rangle$. On the other hand, from Sect. 2, one has

$$
\delta\left(\frac{\partial H}{\partial p}\right)^{-1}=\delta \frac{1}{G}+\frac{\partial N(H, q)}{\partial H},
$$

so that $N(H, q) \neq 0$ in general. This implies that the rotational transform profile $\eta(I)=$ const requires a more general Hamiltonian than $H=p G(q)$ if the configuration is to satisfy the Grad-Shafranov equation. This result is valid for other profiles $\eta(I)$ as well: a particular Hamiltonian $H(p, q)$ which produces a desired $\eta(I)$ is in general not good enough to encompass a solution to the GradShafranov equation.

It is of course possible to obtain axisymmetric MHD equilibria with desired $p(I), \eta(I)$ without bothering about a Hamiltonian. As indicated above, (3.10a), (4.4) combine into an integro-differential 
equation for $I$ which finally yields $F(I)$. The magnetic field follows from (3.8). This approach was used in [13] for the description of "flux conserving tokamaks". Fixed toroidal and poloidal magnetic fluxes also encompass fixed $\eta(I)$. The integro-differential equation was solved approximately in [13], but we shall not further discuss it here. Incidentally, $\eta(I)=$ const as in example 3 was assumed in [13].

\section{Example 2:}

The particular magnetic and current density surfaces which were obtained in Section 3 for this example were constructed starting inwards from a boundary surface $r^{1}=r_{0}^{1}=I=S\left(r^{2}, r^{3}\right)=$ const which also determines the coordinate system and the metric coefficients $g^{i j}\left(r^{1}, r^{2}, r^{3}\right)$. The MHD equilibrium condition, (4.1), (4.2), again requires that $[\boldsymbol{j} \times \boldsymbol{B}]_{1}$, which is a functional of $F(I), G\left(r^{2}, r^{3}\right)$, $S\left(r^{2}, r^{3}\right)$, depend on $I=r^{1}$ only. In view of the many terms which compose $[\boldsymbol{j} \times \boldsymbol{B}]_{1}$ (see (4.5) for the much simpler case of axisymmetry) it seems virtually impossible, however, to select $G\left(r^{2}, r^{3}\right)$ and $S\left(r^{2}, r^{3}\right)$ in such a way that the dependence on $r^{2}$ and $r^{3}$ completely disappears. This is particularly so because it is probably only possible to give the $g^{i j}$ explicitly in terms of $S\left(r^{2}, r^{3}\right)$ for special cases at most.

In consequence, just as in the axisymmetric case, the Hamiltonian method with fixed Hamiltonian and restricted magnetic surfaces is unsuited in practice to proceeding further from configurations with equal magnetic and current density surfaces to MHD equilibria.

\section{Conclusions}

The equations for magnetic field lines are equivalent to a Hamiltonian system. This has been used to obtain a representation of magnetic fields in terms of their toroidal magnetic surfaces $I=$ const, which may be arbitrary, and of arbitrary Hamiltonians $H(p, q, t)$ with appropriate invariants $I_{H}(p, q, t)$. The Hamiltonian uniquely determines the rotational transform $\eta(I)$, but not vice versa. For the general axisymmetric field, for a particular class of nonaxisymmetric fields with constant $\eta(I)$, and for the combination of both classes explicit expressions are given for the magnetic field $\boldsymbol{B}$ and for $\eta(I)$.
For the axisymmetric case configurations with coinciding $\boldsymbol{B}$-surfaces and $\boldsymbol{j}$-surfaces are given explicitly, while for the nonaxisymmetric examples it is shown qualitatively that a particular type of corrugation should make the $\boldsymbol{B}$ - and $\boldsymbol{j}$-surfaces coincide.

The step onwards from necessary equilibrium conditions to a full equilibrium solution, however, proves to be unfeasible. The reason is that in order to make the pressure be a surface function, $H(p, q, t)$ should not be fixed a priori: the above-mentioned freedom in $H$ for given $\eta(I)$ is essential to satisfy the pressure balance. With the Hamiltonian not fixed a priori, however, nothing is known about invariants of $H$ in general, and the method used breaks down. This deficiency is not causally related to the well-known uncertainty whether nonaxisymmetric equilibria exist at all, since it already occurs in the axisymmetric case. It is unlikely that the situation is different for the Hamiltonian system in [3], which differs from the one used here in that, for example, the toroidal flux is treated as the canonical momentum.

In spite of the shortcomings mentioned, the Hamiltonian approach is nevertheless useful for the equilibrium problem. In [14], for example, other aspects of this approach were used successfully to minimize deviations from a true MHD equilibrium.

\section{Acknowledgement}

The author would like to thank $\mathrm{H}$. Tasso, D. Pfirsch, and H. K. Wimmel for helpful discussions.

\section{Appendix}

It is easily seen that the Hamiltonian (2.25) is equivalent to a two-dimensional time-independent Hamiltonian system

$H\left(p_{1}, p_{2}, q_{1}, q_{2}\right)=p_{1} G_{1}\left(q_{1}, q_{2}\right)+p_{2} G_{2}\left(q_{1}, q_{2}\right)$ (A. 1$)$

with $G_{2} \equiv 1$. For Hamiltonians with arbitrary $2 \pi$ periodic $G_{1}, G_{2}$ one has

$$
\operatorname{det}\left\{\frac{\partial^{2} H_{0}}{\partial p_{i} \partial p_{j}}\right\}=0,
$$

where $H_{0}$ is any part of $H$. In this case the theorem of Kolmogorov, Arnold, and Moser (KAM) [15] cannot be applied. For numerical studies see [9]. 
[1] K. J. Whiteman, Rep. Prog. Phys. 40, 1033 (1977).

[2] J. R. Cary and R. G. Littlejohn, Ann. Phys. 151, 1 (1983).

[3] A. H. Boozer, Magnetic Field Line Hamiltonian, Report Princeton PPPL - 2094 (1984).

[4] H. Goldstein, Classical Mechanics, Addison-Wesley, Reading, Mass., 1980.

[5] H. R. Lewis, Jr., J. Math. Phys. 9, 1976 (1968).

[6] J. R. Ray and J. L. Reid, Phys. Lett. 71 A, 317 (1979).

[7] R. S. Kaushal and H. J. Korsch, J. Math. Phys. 22, 1904 (1981).

[8] W. Sarlet and J. R. Ray, J. Math. Phys. 22, 2504 (1981).
[9] A. Salat, Z. Naturforsch. 39a, 830 (1984).

[10] K. Miyamoto, Plasma Physics for Nuclear Fusion, MIT Press, Cambridge, Mass., 1980.

[11] H. Grad and H. Rubin, in Proc. of the Second International Conference on the Peaceful Uses of Atomic Energy (United Nations, Geneva, 31, 190 (1958).

[12] V. D. Shafranov, Sovjet Phys. JETP 6, 545 (1958)

[13] J. F. Clarke and D. J. Sigmar, Phys. Rev. Lett. 38, 70 (1977).

[14] J. D. Hanson and J. R. Cary, Phys. Fluids 27, 767 (1984).

[15] V. I. Arnold, Mathematical Methods of Classical Mechanics, Springer Verlag, New York 1980. 